\title{
FUNCTOR HOMOLOGY AND HOMOLOGY OF COMMUTATIVE MONOIDS
}

\author{
REVAZ KURDIANI AND TEIMURAZ PIRASHVILI
}

The aim of this work is to clarify the realtionship between homology theory of commutative monoids constructed á la Quillen [16], [17] and technology of $\Gamma$ modules as it was developped in [12],[13], [14], [15].

In Section 1 we recall the basics of $\Gamma$-modules and relation with commuttaive algebra (co)homology. In Section 2 we construct an analogue of Kähler differentials for commutative monoids. In Section 3 we construct homology theory for commutative monoids which and then we prove that commutative monoid homology are particular case of functor homology develiped in [13].

It should be pointout that the cohomology theory of commutative monoids first was constructed by P.-A. Grillet in the series of papers [2]-[7] (see also a recent work [1]). So our results shed light on Grillet theory. For instance we relate the commutative monoid (co)homology with André-Quillen (co)homology of corresponding monoid algebra. For another application we mention the Hodge decomposition for commutative monoid (co)homology which is an immediatle consequence of our main result.

\section{1. $\Gamma$-modules And COMmutative Algebra (CO)homology}

1.1. Generalities on $\Gamma$-modules. Let $K$ be a commutative ring with unit which is fixed in the whole paper.

For any integer $n \geq 0$, we let $[n]$ be the set $\{0,1, \ldots, n\}$ with basepoint 0 . Let $\Gamma$ be the full subcategory of the category of pointed sets consisting of sets $[n]$. A left $\Gamma$-module is a covariant functor from $\Gamma$ to the category of $K$-modules, while a right $\Gamma$-module is a contravariant functor from $\Gamma$ to the category of $K$-modules. The category of all left $\Gamma$-modules is denoted by $\Gamma$ - $\bmod$, while the category of all right modules is denoted by $\bmod -\Gamma$. It is well-known that the categories $\Gamma-\bmod$ and mod- $\Gamma$ are abelian categories with sufficiently many projective and injective objects. For any $n \geq 0$ one defines the left $\Gamma$ module $\Gamma^{n}$ by

$$
\Gamma^{n}(X)=K\left[X^{n}\right]
$$

Here $K[S]$ denotes the free $K$-module generated by a set $S$. It is a consequence of the Yoneda lemma that for any left $\Gamma$-module $F$ one has natural isomorphisms

$$
\operatorname{Hom}_{\Gamma}\left(\Gamma^{n}, F\right) \cong F([n]) \text {. }
$$

Therefore $\Gamma^{n}, n \geq 0$, are projective generators of the category $\Gamma$ - $\bmod$.

1.2. Hochschild and Harrison (co)homology of $\Gamma$-modules. The definition of these objects are based on the following pointed maps (see [9]). For any $0 \leq i \leq$ $n+1$, one defines a map

$$
\epsilon^{i}:[n+1] \rightarrow[n], 0 \leq i \leq n+1,
$$

Research was partially supported by the GNSF Grant ST08/3-387. The second author acknowledgement the support given by the University of Leicester in granting academic study leave. 
by

$$
\epsilon^{i}(j)= \begin{cases}j & j \leq i, \\ j-1 & j>i \leq n, \\ 0 & j=i=n+1 .\end{cases}
$$

For a left $\Gamma$-module $F$ the Hochschild homology $H H_{*}(F)$ is defined as the homology of the chain complex

$$
F([0]) \leftarrow F([1]) \leftarrow F([2]) \leftarrow \cdots \leftarrow F([n]) \leftarrow \cdots
$$

where the boundary map $\partial: F([n+1]) \rightarrow F([n])$ is given by $\sum_{i=0}^{n+1}(-1)^{i} F\left(\epsilon^{i}\right)$.

Quite similarly for a right $\Gamma$-module $T$ one defines the Hochschild cohomology $H H^{*}(T)$ as the cohomology of the following cochain complex

$$
T([0]) \rightarrow T([1]) \rightarrow \cdots \rightarrow T([n]) \rightarrow T([n+1]) \rightarrow \cdots
$$

where the coboundary map $\delta: T([n]) \rightarrow T([n+1])$ is given by $\delta=\sum_{i=0}^{n+1}(-1)^{i} T\left(\epsilon^{i}\right)$.

We have the following obvios fact.

Lemma 1.1. Let $F$ be a left $\Gamma$-module, then $H H_{0}(F)=F([0])$ and

$$
H H_{1}(F)=\operatorname{Coker}(\partial: F([2]) \rightarrow F([1])) \text {. }
$$

Similraly, if $T$ is a r ight $\Gamma$-module, then $H H^{0}(T)=T([0])$ and

$$
H H^{1}(T)=\operatorname{ker}(\delta: T([1]) \rightarrow T([2]))
$$

Let $S_{n}$ be the symmetric group on $n$ letters, it acts as a group of automorphisms on $[n]$. For integers $p_{1}, \cdots, p_{k}$ with $n=p_{1}+\cdots+p_{k}$, we set

$$
s h_{p_{1}, \cdots, p_{k}}=\sum \operatorname{sgn}(\sigma) \sigma \in \mathbb{Z}\left[S_{n}\right]
$$

where $\sigma \in S_{n}$ is running over all $\left(p_{1}, \cdots, p_{k}\right)$-shuffles. Each $s h_{p_{1}, \cdots, p_{k}}$ induces a map $T([n]) \rightarrow T([n])$, called the shuffle map. Let us denote by $\tilde{T}_{n}$ the intersection of the kernels of all shuffle maps. These groups form a subcomplex of the Hochschild complex, called Harrison complex [9]. The groups $\operatorname{Harr}^{n}(T), n \geq 0$ are defined as the cohomology of the Harrison complex.

By duality we have also Harrison and Hochschild homology of left $\Gamma$-module.

The following is a theorem due to J.-L. Loday [9]. For alternative approach see [12].

Theorem 1.2. If $K$ is a field of characteristic zero, then for any left $\Gamma$-module $F$ and right $\Gamma$-module $T$ there exist so called Hodge decompositions:

$$
\begin{gathered}
\left.H H_{n}(F) \cong \bigoplus_{i=1}^{n} H H_{n}^{(i)} F\right), \quad n>0, \\
H H^{n}(F) \cong \bigoplus_{i=1}^{n} H H_{(i)}^{n}(T), \quad n>0,
\end{gathered}
$$

for suitable defined $H H_{n}^{(i)}(F)$ and $H H_{(i)}^{n}(T)$. Moroeover, for $i=1$ one has

$$
\operatorname{Harr}_{n}(F)=H H_{n}^{(1)}(F), \operatorname{Harr}^{n}(F)=H H_{(1)}^{n}(T), \quad n>0 .
$$


1.3. André-Quillen (co)homology of $\Gamma$-modules. We recall some material from [13].

A partition $\lambda=\left(\lambda_{1}, \cdots, \lambda_{k}\right)$ is a sequence of natural numbers $\lambda_{1} \geq \cdots \geq \lambda_{k} \geq 1$. The sum of partition is given by $s(\lambda):=\lambda_{1}+\cdots+\lambda_{k}$, while the group $\Sigma(\lambda)$ is a product of the corresponding symmetric groups

$$
\Sigma(\lambda):=\Sigma_{\lambda_{1}} \times \cdots \times \Sigma_{\lambda_{k}}
$$

which is identified with the Young subgroup of $\Sigma_{s(\lambda)}$. Let us observe that $\Sigma_{n}=$ $\operatorname{Aut}_{\Gamma}([n])$ and therefore $\Sigma_{n}$ acts on $F([n])$ and $T([n])$ for any left $\Gamma$-module $F$ and right $\Gamma$-module $T$.

Let

$$
0 \rightarrow F_{1} \rightarrow F \rightarrow F_{2} \rightarrow 0
$$

be an exact sequence of left $\Gamma$-modules. It is called a $\mathcal{Y}$-exact sequence if for any partition $\lambda$ with $s(\lambda)=n$ the induced map

$$
F([n])^{\Sigma(\lambda)} \rightarrow F_{2}([n])^{\Sigma(\lambda)}
$$

is surjective. The class of $\mathcal{Y}$-exact sequences is proper in the sense of MacLane [11].

A left $\Gamma$-module $F$ is $\mathcal{Y}$-projective, if the functor $\operatorname{Hom}_{\Gamma}(F,-)$ takes $\mathcal{Y}$-exact sequences to exact sequences. For example $S^{n} \Gamma^{1}$ is a $\mathcal{Y}$-projective [13]. Here $S^{n}$ denotes the $n$-th symmetric power. According to [13] for any left $\Gamma$-module $F$ there is a $Y$-exact sequence

$$
0 \rightarrow F_{1} \rightarrow F_{0} \rightarrow F \rightarrow 0
$$

with $\mathcal{Y}$-projective $F$. Hence one can take relative left derived functors of the functor $H H_{1}: \Gamma$ - $\bmod \rightarrow K$-mod. The values of these derived functors on a left $\Gamma$-module $F$ is denoted by $\pi_{*}^{\mathcal{Y}}(F)$. So by the definition the functors $\pi_{*}^{\mathcal{Y}}$ are uniquely defined (up to isomorphism) by the following properties

Lemma 1.3. There exist unique family of functors $\pi^{\mathcal{Y}}{ }_{n}: \Gamma-\bmod \rightarrow K-\bmod , n \geq 0$, such that

i) $\pi^{\mathcal{Y}}{ }_{0}(F)=H H_{1}(F)$.

ii) For any $\mathcal{Y}$-exact sequence

$$
0 \rightarrow F_{1} \rightarrow F \rightarrow F_{2} \rightarrow 0
$$

there is a long exact sequence

$$
\cdots \rightarrow \pi^{\mathcal{Y}}{ }_{n+1}\left(F_{2}\right) \rightarrow \pi^{\mathcal{Y}}{ }_{n}\left(F_{1}\right) \rightarrow \cdots \rightarrow \pi^{\mathcal{Y}}{ }_{0}\left(F_{1}\right) \rightarrow \pi^{\mathcal{Y}}{ }_{0}(F) \rightarrow \pi^{\mathcal{Y}}{ }_{0}\left(F_{2}\right) \rightarrow 0 .
$$

iii) The functor $\pi^{\mathcal{Y}}{ }_{n}$ vanishes on $\mathcal{Y}$-projective objects, $n \geq 1$.

By a dual argument for any right $\Gamma$-module $T$ one obtains $K$-modules $\pi_{\mathcal{Y}^{*}}(T)$.

1.4. $\Gamma$-modules and commutative algebras. The classical Hochschild cohomology (as well as the Harrison or Andre-Quillen (co)homology) of commuttaive algebas is a particular case of the cohomology of $\Gamma$-modules [9], [12], [13]. We recall the corresponding results. Let $R$ be a commutative $K$-algebra and $A$ be an $R$-module. We have a left and right $\Gamma$-modules $\mathcal{L}_{*}(R, A)$ and $\mathcal{L}^{*}(R, A)$ defined on objects by

$$
\mathcal{L}^{*}(R, A)([n]):=\operatorname{Hom}\left(R^{\otimes n}, A\right), \quad \mathcal{L}_{*}(R, A)([n]):=R^{\otimes n} \otimes A .
$$

For a pointed map $f:[n] \rightarrow[m]$, the action of $\mathcal{L}^{*}(R, A)$ on $f$ is given by

$$
f^{*}(\psi)\left(a_{1} \otimes \cdots \otimes a_{n}\right)=b_{0} \psi\left(b_{1} \otimes \cdots \otimes b_{m}\right)
$$

while for the functor $\mathcal{L}_{*}(R, A)$ one has:

$$
f_{*}\left(a_{0} \otimes \cdots \otimes a_{n}\right)=b_{0} \otimes \cdots \otimes b_{m}
$$

where $b_{j}=\prod_{f(i)=j} a_{i}, j=0, \cdots, n$.

Then one has [9]:

$$
H H_{*}\left(\mathcal{L}_{*}(R, A)\right)=H H_{*}(R, A), \quad H H^{*}\left(\mathcal{L}^{*}(R, A)\right)=H H^{*}(R, A),
$$




$$
\operatorname{Harr}_{n}\left(\mathcal{L}_{*}(R, A)\right)=\operatorname{Harr}_{m}(R, A), \operatorname{Harr}^{n}\left(\mathcal{L}^{*}(R, A)\right)=\operatorname{Harr}^{m}(R, A) .
$$

where $H_{*}(R, A)$ and $\operatorname{Harr}_{*}(R, A)$ (resp. $\left.H H^{*}(R, A), \operatorname{Harr}^{*}(R, A)\right)$ are the Hochschild and Harrison (co)homology of $R$ with coefficients in $A$.

By [13] a similar result is aslo true for André-Quillen (co)homology of commutative rings. In order to state this result, let us first recall the definition of André-Quillen (co)homology [17].

Let $\mathbf{S C R}$ be category of simplicial commutative rings and let $\mathbf{S S}$ be the category of simplicial sets and let $U: \mathbf{S C R} \rightarrow \mathbf{S S}$ be a forgetful functor. According to [16] there is a unique closed model category structure on the category SCR such that a morphism $f: X_{*} \rightarrow Y_{*}$ of $\mathbf{S C R}$ is weak equivalence (resp. fibration) if $U(f)$ is a weak equivalence (resp. fibration) of simplicial sets. A simplicial commutative ring $X_{*}$ is called free if each $X_{n}$ is a free commutative ring with a base $S_{n}$, such that degeneracy operators $s_{i}: X_{n} \rightarrow X_{n+1}$ maps $S_{n}$ to $S_{n+1}, 0 \leq i \leq n$. Thanks to [16] any free simplicial commutative ring is cofibrant and any cofibrant object is a retract of a free simplicial commutative ring.

We let $C^{*}\left(V^{*}\right)$ be the cochain complex associated to a cosimplicial abelian group $V^{*}$. Let $R$ be a commutative ring and let $A$ be an $R$-module. Then the AndréQuillen cohomology of $R$ with coefficients in $A$ is defined by (see [17]):

$$
\mathrm{D}^{*}(R, A):=H^{*}\left(C^{*}\left(\operatorname{Der}\left(P_{*}, A\right)\right)\right)
$$

where $P_{*} \rightarrow R$ is a cofibrant replacement of the ring $R$ considered as a constant simplicial ring and Der denotes the abelian group of all $K$-derivations.

The André-Quillen homology of $R$ with coefficients in $A$ is defined by

$$
\mathrm{D}_{*}(R, A):=H_{*}\left(C_{*}\left(A \otimes_{P_{*}} \Omega_{P_{*}}^{1}\right)\right),
$$

where $\Omega_{R}^{1}$ is the Kähler differentials of a commutative $K$-algebra $R$.

The main result of [13] claims that there are natural isomorphisms

$$
\pi_{*}^{\mathcal{Y}}\left(\mathcal{L}_{*}(R, A)\right)=D_{*}(A, M), \quad \pi_{\mathcal{Y}}^{*}\left(\mathcal{L}^{*}(R, A)\right)=D^{*}(A, M) .
$$

\section{The category $\mathcal{H}(C)$ associated to a commutative monoid $C$}

2.1. Definition. Let $C$ be a commutative monoid. Define the category $\mathcal{H}(C)$ as follows. Objects of $\mathcal{H}(C)$ are elements of $C$. A morphism from an element $a \in C$ to an element $b$ is a pair $(c, a)$ of elements of $C$ such that $b=c a$. To simplify notations we write $a \stackrel{c}{\rightarrow} a c$ for a morphism $(a, c): a \rightarrow b=a c$. If $: a \stackrel{c}{\rightarrow} a c$ and $a c \stackrel{d}{\rightarrow} a c d$ are morphisms in $\mathcal{H}(C)$, then the composite of these morphisms in $\mathcal{H}(C)$ is $a \stackrel{c d}{\longrightarrow}$ acd.

It is clear that $1 \in C$ is an initial object of $\mathcal{H}(C)$.

A left $\mathcal{H}(C)$-module is a covariant functor $A: \mathcal{H}(C) \rightarrow \mathrm{Ab}$, similarly a right $\mathcal{H}(C)$-module is a contravariant functor $A: \mathcal{H}(C)^{o p} \rightarrow \mathrm{Ab}$.

We let $\mathcal{H}(C)$-mod be the category of left $\mathcal{H}(C)$-modules, while mod- $\mathcal{H}(C)$ denotes the category of right $\mathcal{H}(C)$-modules. If $M$ is a left $\mathcal{H}(C)$-module. Then the value of $M$ on the element $a \in C$ (considered as object of $\mathcal{H}(C)$ ) is denoted by $M(a)$. Moreover if $a, b, c \in C$ and $b=c a$, then we have an induced map $c_{*}: M(a) \rightarrow M(b)$, with obvious properties $1_{*}=\mathrm{Id}$ and $\left(c_{1} c_{2}\right)_{*}=c_{1 *} c_{2 *}$.

Quite similarly, if $N$ is a right $\mathcal{H}(C)$-module, then the value of $N$ on the element $a \in C$ is denoted by $N(a)$. Moreover if $a, b, c \in C$ and $b=c a$, then we have an induced map $c^{*}: N(b) \rightarrow N(a)$, with obvious properties $1^{*}=\mathrm{Id}$ and $\left(c_{1} c_{2}\right)^{*}=c_{2}^{*} c_{1}^{*}$.

The categories $\mathcal{H}(C)$-mod and $\bmod -\mathcal{H}(C)$ are abelian categories with enough projective and injective objects. For any element $a$ of $C$ we let $C^{a}$ and $C_{a}$ be respectively the left and right $\mathcal{H}(C)$-modules defined by

$$
C^{a}(x)=\bigoplus_{c \in(x: a)} \mathbb{Z}, C_{a}(x)=\bigoplus_{c \in(a: x)} \mathbb{Z}
$$


Here for elements $a, b \in C$ we let $(b: a)$ be the set of all elements $c \in C$ such that $b=a c$. By Yoneda lemma for any left $\mathcal{H}(C)$-module $A$ and for any right $\mathcal{H}(C)$-module $B$ one has isomorphisms

$$
\left.\operatorname{Hom}_{\mathcal{H}(C)}\left(C^{a}, A\right) \cong A(a), \quad \operatorname{Hom}_{\mathcal{H}(C)}\left(C_{a}, B\right) \cong B(a)\right) .
$$

It follows that $C^{a}, a \in C$ form a family of projective generators of the category $\mathcal{H}(C)$-mod. Similarly $C_{a}, a \in C$ form a family of projective generators of the category $\bmod -\mathcal{H}(C)$.

Let $N$ be a right $\mathcal{H}(C)$-module and $M$ be a left $\mathcal{H}(C)$-module. We let $N \otimes_{\mathcal{H}(C)} M$ be the abelian group generated by elements of the form $x \otimes y$, where $x \in N(a)$, $y \in M(a), a \in M$. These elements are subject to the following relations

$$
\begin{gathered}
\left(x_{1}+x_{2}\right) \otimes y=x_{1} \otimes y+x_{2} \otimes y, \\
x \otimes\left(y_{1}+y_{2}\right)=x \otimes y_{1}+x \otimes y_{2}, \\
c^{*}(z) \otimes y=z \otimes c_{*}(y) .
\end{gathered}
$$

Here $c \in M, x, x_{1}, x_{2} \in N(a), y, y_{1}, y_{2} \in M(a), z \in N_{c a}$. Then one has

$$
N \otimes_{\mathcal{H}(C)} C^{a} \cong N(a), \quad C_{a} \otimes_{\mathcal{H}(C)} M \cong M(a)
$$

If $f: C \rightarrow C^{\prime}$ is a homomorphism of monoids, then $f$ induces a functor $\mathcal{H}(f)$ : $\mathcal{H}(C) \rightarrow \mathcal{H}\left(C^{\prime}\right)$ in an obvious way. Thus for any left $\mathcal{H}\left(C^{\prime}\right)$-module $M$ one has a left $\mathcal{H}(C)$-module $f^{*}(M)$, which is given by

$$
f^{*}(M)(i)=M(f(i)) \text {. }
$$

In this way one obtains a functor $f^{*}$ from the category of (left or right) modules over $\mathcal{H}\left(C^{\prime}\right)$ to the category of modules over $\mathcal{H}(C)$.

2.2. $K[C]$-modules and $\mathcal{H}(C)$-modules. We let $K[C]$ be the monoid algebra of the monoid $C$. Any $K[C]$-module $A$ gives rise to the left $\mathcal{H}(C)$-module $j^{*}(A)$ which is defined by

$$
j^{*}(A)(a)=A
$$

and for $b=c a$, the induced morphisms

$$
A=j_{*}(A)(a) \stackrel{c_{*}}{\longrightarrow} j_{*}(A)(b)=A
$$

is simply the multiplication by $c$.

If $M$ is a left $\mathcal{H}(C)$-module, we let $j_{*}(M)$ be the following $K[C]$ module. As an abelian group one has

$$
j_{*}(M)=\bigoplus_{x \in C} M(x)
$$

The module structure is defined as follows: for $x \in C, a \in M(x)$ and $c \in C$ one has

$$
c i_{x}(a)=i_{c x}\left(c_{*}(a)\right) .
$$

Here $i_{x}$ is the canonical inclusion $M(x) \rightarrow j_{*}(M), x \in C$.

Lemma 2.1. The functor $j_{*}$ is a left adjoint functor to $j^{*}$.

Proof. For a left $\mathcal{H}(C)$-module $M$ and a left $K[C]$-module $A$ an elements

$$
\xi \in \operatorname{Hom}_{\mathcal{H}(C)}\left(M, j^{*}(A)\right)
$$

is given by the family of $K$-module homomorphisms $\xi_{a}: M(a) \rightarrow A, a \in A$ such that for any $c \in C$ the following

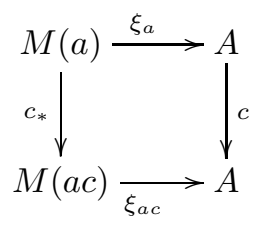


The homomorphisms $\xi_{a}, a \in C$ defines a homomorphism of $K$-modules

$$
\hat{\xi}: j_{*}(M)=\bigoplus_{a \in C} M(a) \rightarrow A
$$

which clearly is $K[M]$-homomorphism. So, $\xi \mapsto \hat{\xi}$ gives rise a homomorphism $\operatorname{Hom}_{\mathcal{H}(C)}\left(M, j^{*}(A)\right) \rightarrow \operatorname{Hom}_{K[C]}\left(j_{*}(M), A\right)$ which is obviously an isomorphism.

2.3. Derivations, differentials and (co)homology in the theory of commutative algebras. Let $C$ be a commutative monoid and let $M$ be a left $\mathcal{H}(C)$ module. A derivation $\delta: C \rightarrow M$ of $C$ with values in $M$ is a function which assigns to each element $a \in C$ an element $\delta(a) \in M(a)$, such that

$$
\delta(a b)=a_{*}(\delta(b))+b_{*}(\delta(a)) .
$$

The abelian group of all derivations of $C$ with values in $M$ is denoted by $\operatorname{Der}(C, M)$.

We claim that there exist a universal derivation. In fact we construct a left $\mathcal{H}(C)$ module $\Omega_{C}$, called differentials of a monoid $C$. It is a left $\mathcal{H}(C)$-module generated by symbols $d a \in \Omega_{C}(a)$ one for every element $a \in C$, subject to relations

$$
d(a b)=a_{*}(d(b))+b_{*}(d(a))
$$

for every $a$ and $b \in C$. It follows from the construction that $a \mapsto d a$ is a derivation, which is clearly universal one, in the sense that for any derivation $\delta: C \rightarrow, M$ there is a unique homomorphism of $\mathcal{H}(C)$-modules $\delta^{*}: \Omega_{C} \rightarrow M$ such that $\delta(a)=\delta^{*}(d a)$. Thus for any left $\mathcal{H}(C)$-module $M$ one has a canonical isomorphism

$$
\operatorname{Der}(C, M) \cong \operatorname{Hom}_{\mathcal{H}(C)}\left(\Omega_{C}, M\right) \text {. }
$$

Lemma 2.2. One has an isomorphism of $K[C]$-modules

$$
j_{*}\left(\Omega_{C}\right)=\Omega_{K[C]}^{1}
$$

Here $j_{*}: \mathcal{H}(C)$-mod $\rightarrow K[C]$-mod is the functor constructed in Section 2.2 and $\Omega_{K[C]}^{1}$ is the Kähler differentials of the $K$-algebra $K[C]$.

Proof. Let $A$ be a $K[C]$-module. Then we have

$$
\operatorname{Der}\left(C, j^{*}(A)\right)=\operatorname{Hom}_{\mathcal{H}(C)}\left(\Omega_{C}, j^{*}(A)\right)=\operatorname{Hom}_{K[C]}\left(j_{*}(\Omega), A\right) .
$$

On the other hand

$$
\operatorname{Der}\left(C, j^{*}(A)\right)=\operatorname{Der}(K[C], A)=\operatorname{Hom}_{K[C]}\left(\Omega_{K[A]}^{1}, A\right)
$$

and the result follows from the Yoneda lemma.

2.4. The case $C=\mathbb{N}$. If $C$ is the free abelian monoid with a generator $t$, then a left $\mathcal{H}(C)$-module is nothing but a diagram of abelian groups

$$
M=\left(M_{0} \stackrel{t}{\rightarrow} M_{1} \stackrel{t}{\rightarrow} M_{2} \stackrel{t}{\rightarrow} M_{3} \stackrel{t}{\rightarrow} \cdots\right)
$$

In particular the projective object $C^{n}$ corresponds to the diagram

$$
0 \rightarrow 0 \rightarrow \cdots \rightarrow 0 \rightarrow \mathbb{Z} \stackrel{1}{\rightarrow} \mathbb{Z} \stackrel{1}{\rightarrow} \cdots
$$

where the first nontrivial group appears at the place $n$.

Quite similarly a right $\mathcal{H}(C)$-module is nothing but a diagram of abelian groups

$$
N=\left(\cdots \stackrel{t}{\rightarrow} N_{3} \stackrel{t}{\rightarrow} N_{2} \stackrel{t}{\rightarrow} N_{1} \stackrel{t}{\rightarrow} N_{0}\right) .
$$

In particular the projective object $C_{n}$ corresponds to the diagram

$$
\cdots \rightarrow 0 \rightarrow 0 \rightarrow \mathbb{Z} \stackrel{1}{\rightarrow} \mathbb{Z} \stackrel{1}{\rightarrow} \cdots \stackrel{1}{\rightarrow} \mathbb{Z}
$$

where the first nontrivial group appears at the place $n$.

One easily observes that for any left $\mathcal{H}(C)$-module $M$ one has an isomorphism

$$
\operatorname{Der}(C, M) \cong M_{1}
$$


which is given by $\delta \mapsto \delta(t)$. This follows from the fact that $\delta\left(t^{n}\right)=n t^{n-1} \delta(t)$. Thus

$$
\Omega_{C}=C^{1}=(0 \rightarrow \mathbb{Z} \stackrel{1}{\rightarrow} \mathbb{Z} \stackrel{1}{\rightarrow} \mathbb{Z} \stackrel{1}{\rightarrow} \cdots) .
$$

2.5. Product of two monoids. Let $C$ be a product of two monoids: $C=C_{1} \times C_{2}$. Then $\mathcal{H}(C)=\mathcal{H}\left(C_{1}\right) \times \mathcal{H}\left(C_{2}\right)$. Assume $M_{1}$ and $M_{2}$ are (say left) $\mathcal{H}\left(C_{1}\right)$ and $\mathcal{H}\left(C_{2}\right)-$ modules respectively. Then one can form a $\mathcal{H}(C)$-module $M_{1} \otimes M_{2}$ as follows:

$$
M_{1} \otimes M_{2}\left(x_{1}, x_{2}\right)=M_{1}\left(x_{1}\right) \otimes M_{2}\left(x_{2}\right) .
$$

Lemma 2.3. For any elements $c_{1} \in C$ and $c_{2} \in C_{2}$, one has

$$
C^{\left(c_{1}, c_{2}\right)}=C^{c_{1}} \otimes C^{c_{2}}
$$

and

$$
C_{\left(c_{1}, c_{2}\right)}=C_{c_{1}} \otimes C_{c_{2}}
$$

Proof. By definition one has

$$
\begin{aligned}
C^{c_{1}} \otimes C^{c_{2}}\left(x_{1}, x_{2}\right) & =C^{c_{1}}\left(x_{1}\right) \otimes C^{c_{2}}\left(x_{2}\right) \\
& =\left(\begin{array}{c}
\bigoplus \\
a_{1} \in C_{1} ; a_{1} c_{1}=x_{1}
\end{array}\right) \otimes\left(\bigoplus_{a_{2} \in C_{2} ; a_{2} c_{2}=x_{2}} \mathbb{Z}\right) \\
= & \bigoplus \bigoplus_{\left(a_{1}, a_{2}\right)\left(c_{1}, c_{2}\right)=\left(x_{1}, x_{2}\right)} \mathbb{Z} \\
= & C^{\left(c_{1}, c_{2}\right)}\left(x_{1}, x_{2}\right) .
\end{aligned}
$$

Similarly for the second isomorphism.

We have homomorphisms

$$
\iota_{1}: C_{1} \rightarrow C, \iota\left(c_{1}\right)=\left(c_{1}, 1\right), \quad \iota_{2}: C_{2} \rightarrow C, \iota\left(c_{2}\right)=\left(1, c_{2}\right) .
$$

For any left $\mathcal{H}(C)$-module $M$ we set

$$
M^{(1)}=\iota_{1}^{*}(M), \quad M^{(2)}=\iota_{2}^{*}(M) .
$$

Lemma 2.4. For any left $\mathcal{H}(C)$-module $M$ one has

$$
\operatorname{Der}(C, M) \cong \operatorname{Der}\left(C_{1}, M^{(1)}\right) \oplus \operatorname{Der}\left(C_{1}, M^{(2)}\right) .
$$

Proof. This easily follows from the fact $\left(c_{1}, c_{2}\right)=\left(c_{1}, 1\right)\left(1, c_{2}\right)$.

We also have projections $\pi_{1}: C \rightarrow C_{1}$ and $\mathrm{s} \pi_{2}: C \rightarrow C_{2}$, given respectively by $p i_{i}\left(c_{1}, c_{2}\right)=c_{i}, i=1.2$.

Lemma 2.5. For any left $\mathcal{H}\left(C_{i}\right)$-module $X_{i}, i=1,2$ and any left $\mathcal{H}(C)$-module $M$, one has isomorphisms

$$
\operatorname{Hom}_{\mathcal{H}(C)}\left(\pi_{1}^{*} X_{1}, M\right) \cong \operatorname{Hom}_{\mathcal{H}\left(C_{1}\right)}\left(X_{1}, M^{(1)}\right)
$$

and

$$
\operatorname{Hom}_{\mathcal{H}(C)}\left(\pi_{2}^{*} X_{2}, M\right) \cong \operatorname{Hom}_{\mathcal{H}\left(C_{2}\right)}\left(X_{2}, M^{(2)}\right) .
$$

Proof. Let $\eta \in \operatorname{Hom}_{\mathcal{H}(C)}\left(\pi_{1}^{*} X_{1}, M\right)$. Thus $\eta$ is a collection of homomorphisms of $K$-modules

$$
\eta_{\left(a_{1}, a_{2}\right)}: X_{a_{1}} \rightarrow M_{\left(a_{1}, a_{2}\right)}
$$

such that for any elements $c_{1} \in C_{1}, c_{2} \in C_{2}$ the following diagram commutes

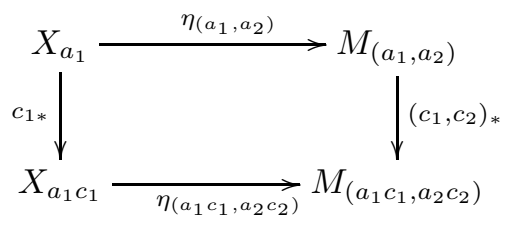


it follows that $\eta_{\left(a_{1}, a_{)}\right.}=\left(1, a_{2}\right)_{*} \circ \eta_{\left(a_{1}, 1\right)}$. It is clear that the familly of homomorphisms $\eta_{a_{1}, 1}, a_{1} \in C_{1}$ defines the morphism $\hat{\eta} \in \operatorname{Hom}_{\mathcal{H}\left(C_{1}\right)}\left(X_{1}, M^{(1)}\right)$ and the previous equality shows that $\eta \mapsto \hat{\eta}$ is really a bijection.

Corollary 2.6. If $C=C_{1} \times C_{2}$, then

$$
\Omega_{C}=\pi_{1}^{*} \Omega_{C_{1}} \oplus \pi_{2}^{*} \Omega_{C_{1}},
$$

where $\pi_{i}: C \rightarrow C_{i}, i=1,2$ is the canonical projection.

Proof. For any $\mathcal{H}(C)$-module $M$ one has

$$
\begin{aligned}
\operatorname{Hom}_{\mathcal{H}(C)}\left(\Omega_{C}, M\right) & =\operatorname{Der}(C, M) \\
& =\operatorname{Der}\left(C_{1}, M^{(1)}\right) \oplus \operatorname{Der}\left(C_{2}, M^{(2)}\right) \\
& =\operatorname{Hom}_{\mathcal{H}\left(C_{1}\right)}\left(\Omega_{C_{1}}, M^{(1)}\right) \oplus \operatorname{Hom}_{\mathcal{H}\left(C_{2}\right)}\left(\Omega_{C_{2}}, M^{(2)}\right) \\
& =\operatorname{Hom}_{\mathcal{H}(C)}\left(\pi_{1}^{*} \Omega_{C_{1}}, M\right) \oplus \operatorname{Hom}_{\mathcal{H}(C)}\left(\pi_{2}^{*} \Omega_{C_{2}}, M\right) \\
& =\operatorname{Hom}_{\mathcal{H}(C)}\left(\pi_{1}^{*} \Omega_{C_{1}} \oplus \pi_{2}^{*} \Omega_{C_{2}}, M\right)
\end{aligned}
$$

and the result follows from the Yoneda lemma.

\section{Commutative monoid (CO)homology and $\Gamma$-modules}

3.1. $\Gamma$-modules related to monoids. Let $C$ be a commutative monoid and let $M$ be a left $\mathcal{H}(C)$-module. Define a right $\Gamma$-module $\mathrm{G}^{*}(C, M)$ as follows. On objects it is igiven by

$$
\mathrm{G}^{*}(C, M)([n])=\prod_{\left(a_{1}, \cdots, a_{n}\right) \in C^{n}} M_{a_{1} \cdots a_{n}} .
$$

Thus $\eta \in \mathrm{G}(C, M)([n])$ is a function which assigns to any $n$-tuple of elements $\left(a_{1}, \cdots, a_{n}\right)$ of $C$ an element $\eta\left(a_{1}, \cdots, a_{n}\right) \in M_{a_{1} \cdots a_{n}}$. Let $f:[n] \rightarrow[m]$ be a pointed map and $\xi \in \mathrm{G}(C, M)([m])$. Then the function $f^{*}(\xi) i \in \mathrm{G}(C, M)([n])$ is given by

$$
f^{*}(\xi)\left(a_{1}, \cdots, a_{n}\right)=b_{0 *}\left(\xi\left(b_{1}, \cdots, b_{m}\right)\right)
$$

Quite similarly, let $N$ be a right $\mathcal{H}(C)$-module. Define left $\Gamma$-module $\mathrm{G}_{*}(C, N)$ as follows. On objects it is given by

$$
\mathrm{G}_{*}(C, N)([n])=\bigoplus_{\left(a_{1}, \cdots, a_{n}\right) \in C^{n}} N\left(a_{1} \cdots a_{n}\right) .
$$

In order, to extend the definition on morphism, we let

$$
\iota_{\left(a_{1}, \cdots, a_{n}\right)}: N\left(a_{1} \cdots a_{n}\right) \rightarrow \mathrm{G}_{*}(C, N)([n])
$$

be the canonical inclusion. Let $f:[n] \rightarrow[m]$ be a pointed map. Then the homomorphism

is defined by

$$
f_{*}: \mathrm{G}_{*}(C, N)([n]) \rightarrow \mathrm{G}_{*}(C, N)([m])
$$

$$
f_{*} \iota_{\left(a_{1}, \cdots, a_{n}\right)}(x)=\iota_{\left(b_{1}, \cdots, b_{m}\right)}\left(\left(b_{0}\right)_{*}(x)\right),
$$

where $x \in N\left(a_{1} \cdots a_{n}\right)$ and

$$
b_{j}=\prod_{f(i)=j} a_{i}, \quad j=0, \cdots, n .
$$

Here we used the convention that $b_{0}=1$ provided $f^{-1}(\{0\})=\{0\}$.

Lemma 3.1. Let $C=\mathbb{N}$ be a free commutative monoid with a generaor $t$, and let $C_{n}$ be the standard projective right $\mathcal{H}(C)$-module, $n \geq 0$, see Section 2.4. Then one has an isomorphism of left $\Gamma$-modules

$$
\mathrm{G}_{*}\left(C, C_{n}\right)=\bigoplus_{k=0}^{n} S^{k} \circ \Gamma^{1}
$$


In particular, $\mathrm{G}_{*}\left(C, C_{n}\right)$ is $\mathrm{Y}$-projective.

Proof. Since $\Gamma^{1}([m])$ is a free $K$-module spanned on $x_{1}, \cdots, x_{m}$, it follows that $S^{k} \circ \Gamma^{1}([m])$ is a free $K$-module spanned by all monomials of degree $k$ on the variables $x_{1}, \cdots, x_{m}$. On the other hand we have

$$
\mathrm{G}_{*}\left(C, C_{n}\right)([m])=\bigoplus_{k=0}^{n} \bigoplus_{n_{1}+\cdots+n_{m}=k} \mathbb{Z} .
$$

To see the expected isomorphism, it is enough to assigne to a basis element of $\bigoplus_{n_{1}+\cdots+n_{k}=m} \mathbb{Z}$ the monomial $x_{1}^{n_{1}} \cdots x_{m}^{n_{m}}$.

Lemma 3.2. Let $C=C_{1} \times C_{2}$ be product of two monoids and $N_{i}$ be right $\mathcal{H}\left(C_{i}\right)$ modules, $i=1,2$. Then one has

$$
\mathrm{G}_{*}\left(C, N_{1} \otimes N_{2}\right)=\mathrm{G}_{*}\left(C_{1}, N_{1}\right) \otimes \mathrm{G}_{*}\left(C_{2}, N_{2}\right) .
$$

The proof is straightforward.

Corollary 3.3. Let $C$ be a finitely generated free commutative monoid and let $N$ be a projective object in the category of right $\mathcal{H}(C)$-modules. Then $\mathrm{G}_{*}(C, N)$ is a $\mathrm{Y}$-projective left $\Gamma$-module.

Proof. Since, any projective object is a retract of a direct sum of standard projective modules $C^{c}$, it is enough to restrict ourself with the case when $C=C^{c}$. Assume $C=\mathbb{N}^{k}$. We will work by induction on $k$. If $k=1$, then the result was already established, see Lemma 3.1. Rest follows from Lemma 2.4 and Lemma 3.2 and the fact that tensor product of two Y-projective objects is $\mathrm{Y}$-projective see [13].

3.2. Homology and cohomology of commutative monoids. Let CM be the category of all commutative monoids and let SCM be the category of all simplicial commutative monoids. There is a forgetful functor $U^{\prime}:$ SCM $\rightarrow$ SS. By [16] there is a unique closed model category structure on the category SCM such that a morphism $f: X_{*} \rightarrow Y_{*}$ of $\mathbf{S C M}$ is a weak equivalence (resp. fibration) if $U^{\prime}(f)$ is a weak equivalence (resp. fibration) of simplicial sets. A simplicial commutative monoid $X_{*}$ is called free if each $X_{n}$ is a free commutative monoid with a base $Y_{n}$, such that degeneracy operators $s_{i}: X_{n} \rightarrow X_{n+1}$ maps $Y_{n}$ to $Y_{n+1}, 0 \leq i \leq n$. According to [16] any free simplicial commutative monoid is cofibrant and any cofibrant object is a retract of a free simplicial commutative monoid.

If $C^{\prime} \rightarrow C$ is a morphism of commutative monoids then it gives rise to a functor $\mathcal{H}\left(C^{\prime}\right) \rightarrow \mathcal{H}(C)$, which allows us to consider any left or right $\mathcal{H}(C)$-module as a module over $\mathcal{H}\left(C^{\prime}\right)$. In particular if $P_{*} \rightarrow C$ is an augmented simplicial monoid and $M$ is a left $\mathcal{H}(C)$-module, one can considered $M$ as a left $\mathcal{H}\left(P_{k}\right)$-module, for all $k \geq 0$. The same holds for right $\mathcal{H}(C)$-modules.

Let $M$ be a left $\mathcal{H}(C)$-module. Then the Grillet cohomology of $C$ with coefficients in $M$ is defined by

$$
\mathrm{D}^{*}(C, M):=H^{*}\left(C^{*}\left(\operatorname{Der}\left(P_{*}, M\right)\right)\right),
$$

where $P_{*} \rightarrow C$ is a cofibrant replacement of the monoid $C$ considered as a constant simplicial monoid.

Let $N$ be a right $\mathcal{H}(C)$-module. Then the Grillet homology of $C$ with coefficients in $N$ is defined by

$$
\left.\mathrm{D}_{*}(C, N):=H_{*}\left(C_{*}\left(\Omega_{P_{*}} \otimes_{\mathcal{H}\left(P_{*}\right)} N\right)\right)\right),
$$

where $P_{*} \rightarrow C$ is a cofibrant replacement of the monoid $C$ considered as a constant simplicial monoid.

The definition of the cohomology essentially goes back to Grillet (see [2]-[5]), but the definition of the Grillet homology is new.

By comparing the definition we obtain the following basic fact, which is missing in (see [2]-[5]). 
Lemma 3.4. Let $C$ be a commutative monoid and $A$ be a $K[C]$-module. Then one has the isomorphisms:

$$
\begin{aligned}
& \mathrm{D}^{*}\left(C, j^{*}(A)\right) \cong \mathrm{D}^{*}(K[C], A), \\
& \mathrm{D}_{*}\left(C, j^{*}(A)\right) \cong \mathrm{D}_{*}(K[C], A) .
\end{aligned}
$$

Proof. The isomorphism in the dimension zero is obvios one, compare with Lemma 2.2. Rest follows from the fact that if $P_{*} \rightarrow C$ is a cofibrant replacement of $C$ in the category SCM, then $K\left[P_{*}\right] \rightarrow K[C]$ is a cofibration replacement of $K[C]$ in the category SCR.

3.3. The main Theorem. Now we are in the situation to state our main theorem, which relates Grillet (co)homology of the monoid $M$ with the Andre-Quillen (co)homology of the $\Gamma$-modules $\mathrm{G}_{*}(C, N)$ and $\mathrm{G}^{*}(C, M)$.

Theorem 3.5. Let $C$ be a commutative monoid, $M$ be a left and $N$ be a right $\mathcal{H}(C)$-modules. Then one has the following isomorphisms

$$
\begin{aligned}
& \mathrm{D}^{*}(C, M)=\pi_{\mathcal{Y}}^{*}\left(\mathrm{G}^{*}(C, M)\right), \\
& \mathrm{D}_{*}(C, M)=\pi^{\mathcal{Y}}{ }_{*}\left(\mathrm{G}_{*}(C, N)\right) .
\end{aligned}
$$

The proof is based on several steps. The idea is to reduce the theorem to the case when $M$ is a free commutative monoid with one generators. In this case, theorem is proved using direct computation. We give proof only for homology, a dual argument works for cohomology.

We need some lemmata.

Lemma 3.6. Let $C$ be a commutative monoid, $N$ be a right $\mathcal{H}(C)$-module. Then one has natural isomorphisms

$$
H H_{1}\left(\mathrm{G}_{*}(C, N)\right) \cong N \otimes_{\mathcal{H}(C)} \Omega_{C} .
$$

Proof. Thanks to Lemma 1.1 one has $H H_{1}\left(\mathrm{G}_{*}(C, N)\right.$ is isomorphic to the cokernel of the map

$$
\partial: \bigoplus_{a, b \in C} N(a b) \rightarrow \bigoplus_{a \in C} N(a)
$$

As usual, we let $i_{a}: N(a) \rightarrow \bigoplus_{a \in C} N(a)$ be the canonical inclusion. For an element $x \in N(a)$, the class of $i_{a}(x)$ in $H H_{1}\left(\mathrm{G}_{*}(C, N)\right)$ is denoted by $c l(a ; x)$. Then

$$
\operatorname{cl}(a ; x) \mapsto x \otimes d a
$$

defines the isomorphism $H H_{1}\left(\mathrm{G}_{*}(C, N)\right) \cong N \otimes_{\mathcal{H}(C)} \Omega_{C}$.

Lemma 3.7. Let $C$ be a commutative monoid and let

$$
0 \rightarrow N_{1} \rightarrow N \rightarrow N_{2} \rightarrow 0
$$

ibe a short exact sequence of right $\mathcal{H}(C)$-modules, then

$$
0 \rightarrow \mathrm{G}_{*}\left(C, N_{1}\right) \rightarrow \mathrm{G}_{*}(C, N) \rightarrow \mathrm{C}_{*}\left(C, N_{2}\right) \rightarrow 0
$$

is a $\mathcal{Y}$-exact sequence of left $\Gamma$-modules.

Proof. For a partition $\lambda$ of $n$ and a set $P$ we denote by $P^{\lambda}$ theset of orbits of the cartesian product $P^{n}$ under the action of the group $\Sigma(\lambda) \subset \Sigma_{n}$. In particular we have a set $C^{\lambda}$. For any element $\mu \in C^{\lambda}$ we put $N_{\mu}:=N\left(a_{1} \cdots a_{n}\right)$, where $\left(a_{1}, \cdots, a_{n}\right) \in \mu$. Since

$$
\mathrm{G}_{*}(C, N)([n])^{\Sigma(\lambda)}=\bigoplus_{\mu \in C^{\lambda}} N_{\mu}
$$

the result follows.

By the same argument we have also the following. 
Lemma 3.8. Let $f: D \rightarrow C$ be a surjective homomorphism of commutative monoids, then for any right $\mathcal{H}(C)$-module $N$ the induced morphism of left $\Gamma$-modules

$$
\mathrm{G}_{*}(D, M) \rightarrow \mathrm{G}_{*}(C, M)
$$

is a $\mathcal{Y}$-epimorphism.

Proof. In the notation of the proof Lemma 3.7 the map $D^{\lambda} \rightarrow C^{\lambda}$ is surjective and the result follows.

Lemma 3.9. Let $\epsilon: X_{*} \rightarrow C$ be a simplicial resolution in the category of commutative monoids and $N$ be a right $\mathcal{H}(C)$-module. Then the associated chain complexes of the simplicial left $\Gamma$-module $\mathrm{G}_{*}\left(X_{*}, N\right) \rightarrow \mathrm{G}_{*}(C, N)$ is a $\mathcal{Y}$-resolution.

Proof. Since $X_{*}^{\lambda} \rightarrow C^{\lambda}$ is a weak equivlence the result follows.

3.4. Proof of Theorem 3.5. Thanks to Lemma 3.6 Theorem is true for $i=0$. First we consider the case, when $C=\mathbb{N}$ is the free commutative monoid with a generator $t$. In this case $\mathrm{D}_{i}(C,-)=0$, if $i>0$. On the other hand $\mathrm{G}_{*}\left(C, C_{n}\right)$ is $\mathcal{Y}$-projective thanks to Lemma 3.1. Therefore the result is true in this case. It follows from Lemma 2.3, Lemma 3.2 and Lemma 4.2 of [13] that the result is true if $C$ is a free commutative monoid and $N$ is projective. By Lemma 3.7 the functor $\pi_{*}^{\mathcal{Y}}\left(\mathrm{G}_{*}(C,-)\right)$ assignes the long exact sequence to a short exact sequence of right $\mathcal{H}(C)$-modules. Therefore we can consider such an exact sequence associated to a short exact sequence of right $\mathcal{H}(C)$-modules

$$
0 \rightarrow N_{1} \rightarrow F \rightarrow N \rightarrow 0
$$

with projective $F$. Since the result is true if $i=0$ one obtains by induction on $i$, that $A Q_{i}\left(\mathrm{G}_{*}(C,-)\right)=0$ provided $i>0$ and $C$ is free commutative monoid.

Now consider the general case. Let $P_{*} \rightarrow C$ be a free simplicial resolution in the category of commutative monoids. Then we have

$$
N \otimes_{\mathcal{H}(P)_{*}} \Omega \cong \pi^{\mathcal{Y}}{ }_{0}\left(\mathrm{G}_{*}(C, N)\right)
$$

Thanks to Lemma $3.9 C_{*}\left(\mathrm{G}_{*}\left(P_{*}, N\right)\right) \rightarrow \mathrm{G}_{*}(C, N)$ is a $\mathcal{Y}$-resolution consisting with $A Q_{*}$-acyclic objects and the result follows.

3.5. Applications. Let $C$ be a commutative monoid, $M$ be a left $\mathcal{H}(C)$-module and $N$ be a right $\mathcal{H}(C)$-module. For the $\Gamma$-modules $\mathrm{G}_{*}(C, N)$ and $\mathrm{G}^{*}(C, M)$ one can apply the reach theory of functor homology developped in [9], [12], [13]. For example if one applies the Hochschild and Harrison theoryies one gets groups $H_{*}(C, N)$, $\operatorname{Harr}_{*}(C, N)$ and $H H^{*}(C, M), \operatorname{Harr}^{*}(C, M)$. Comparing with definitions one sees that $H H^{*}(C, M)$ is nothing but Leech cohomology [8]. If $K$ is a field of characteristic zero, then we have

$$
D_{*}(C, N)=\operatorname{Harr}_{*+1}(C, N), \quad D^{*}(C, M)=\operatorname{Harr}^{*+1}(C, M)
$$

this follows from general results valid for arbitrary $\Gamma$-modules [12],[13]. In particular this solves the cocycle problem for Grillet cohomology [6]. By theorem 1.2 we also obtain that the Grillet cohomology is a direct summand of Leech cohomology.

\section{REFERENCES}

[1] M. Calvo-Cervera, A. M. Cegarra,B. A. Heredia. On the third cohomology group of commutative monoids. arXiv. 1406. 6835.

[2] P. A. Grillet. Commutative semigroup cohomology. Semigroup Forum 43(1991) $247-252$.

[3] P. A. Grillet. The commutative cohomology of nilsemigroups, J. Pure and Appl. Algebra, 82(1992), 233-251.

[4] P. A. Grillet. Commutative semigroup cohomology. Comm. algebra, 23(1995), 35733587. 
[5] P. A. Grillet. The commutative cohomology of finite semigroup. J. Pure and Appl. Algebra, 102(1995), 25-47.

[6] P. A. Grillet. Cocycles in commutative semigroup cohomology. Comm. Algebra 25(1997) 3425-3462.

[7] P. A. GRILlet. The commutative cohomology of two-generator semigroups. Semigroup forum 60(2000), 4-15.

[8] J. LeECH. Two papers: $\mathcal{H}$-coextensions of monoids and the structure of a band of groups. Memoirs A.M.S. 157 (1975)

[9] J. - L. Loday. Opérations sur l'homologie cyclique des algébres commutatives. Invent. math. 96(1989), 205-230.

[10] J. - L. Loday. Cyclic Homology, Grund. Math. Wiss. vol. 301, 2nd edition. Springer, 1998.

[11] S. MacLane. Homology. Classics in Mathematics. Springer-Verlag, Berlin, 1995. x+422 pp.

[12] T. Pirashvili. Hodge decomposition of higher order Hochschild homology. Ann. Sci. École Norm. Sup. (4) 33 (2000), no. 2, 151-179.

[13] T. Pirashvili. André-Quillen homology via functor homology. Proc. AMS 131(2002) 1687-1694.

[14] T. Pirashvili. Dold-Kan type theorem for Г-groups. Math. Ann. 318 (2000), no. 2, 277-298.

[15] T. Pirashvili and B. Richter. Robinson-Whitehouse complex and stable homotopy. topology 29(2000), 525-530.

[16] D. G. QuiLlen. Homotopical algebra. Lecture Notes in Mathematics, No. 43 SpringerVerlag, Berlin-New York 1967 iv+156 pp.

[17] D.G. Quillen. On the (co)homology of commutative rings. AMS Proc. Sym. Pure Math. XVII (1970), 65-87.

Ivane Javakhishyili Tbilisi State University, Georgia

Department of Mathematics, University of Leicester, University Road, Leicester, LE1 7RH, UK

E-mail address: tp59-at-le.ac.uk 